\title{
Neurogenic Bowel, Disordered Glycemic Control and Chronic Spinal Cord Injury: A Preliminary Investigation
}

\author{
Stillman $\mathbf{M}^{1 *}$, Graves $\mathrm{D}^{1}$, Lenneman $\mathrm{C}^{2}$ and \\ Williams $\mathbf{S}^{1}$ \\ ${ }^{1}$ Department of Rehabilitation Medicine, Sidney Kimmel \\ Medical College of Thomas J efferson University, USA \\ ${ }^{2}$ Department of Internal Medicine, University of \\ Louisville School of Medicine, USA \\ *Corresponding author: Stillman M, Departments of \\ Internal Medicine and Rehabilitation Medicine, Sidney \\ Kimmel Medical College of Thomas J efferson University, \\ 1100 Walnut Street, Suite 601, Philadelphia, PA 19107, \\ USA
}

Received: March 20, 2017; Accepted: April 13, 2017; Published: April 20, 2017

\begin{abstract}
Objective/Background: To evaluate potential links between neurogenic bowel and disordered glycemic control in individuals with chronic spinal cord injury $(\mathrm{SCl})$.

Design: Administration of a validated neurogenic bowel questionnaire to participants with $\mathrm{SCl}$, followed by oral glucose tolerance testing (OGTT) and Hemoglobin A1c (HbA1c) assessment in injured subjects and non-injured controls.
\end{abstract}

Methods: Assessment of severity of neurogenic bowel, $\mathrm{HbA1C}$, and performance on OGTT in individuals with $\mathrm{SCl}(\mathrm{n}=19)$ and non-injured controls $(n=10)$.

Outcome Measures: Correlation between classification of severity of neurogenic bowel, $\mathrm{HbA1c}$ levels, and serum glucose and insulin values during a 4-part OGTT.

Results or Findings: Subjects with $\mathrm{SCl}$ had significantly higher insulin levels at 30 minutes and insulin and glucose levels at 60 and 120 minutes than non-injured controls. Average HbA1c levels were no different between the groups (5.52 vs 5.56). Individuals with severe neurogenic bowel had nonsignificant elevations in insulin levels at 30,60 , and 120 minutes and in glucose levels at 120 minutes when compared with those with moderate, mild, or very mild neurogenic bowel.

Conclusion: While not reaching statistical significance, these results may indicate a link between the altered bowel function and post-prandial hyperglycemia of chronic $\mathrm{SCl}$ and indicate a need for further study.

Keywords: Spinal Cord Injury; Hyperglycemia; Neurogenic Bowel

\section{Abbreviations}

SCI: Spinal Cord Injury; DM: Diabetes Mellitus; IGT: Impaired Glucose Tolerance; OGTT: Oral Glucose Tolerance Test; HbAlc: Hemoglobin A1c

\section{Introduction}

Several authors have investigated the prevalence of disordered glycemic control in chronic spinal cord injury (SCI). Duckworth et al found diabetes mellitus (DM) in over half of veterans with SCI undergoing oral glucose tolerance tests (OGTT) [1], Bauman and Spungen described either frank DM or impaired glucose tolerance (IGT) in 56 percent of subjects with SCI [2], and Elder et al conducted OGTT on 12 individuals with injuries, reporting an average 2 hour glucose level of 142, within the range of IGT [3].

Various etiologies of hyperglycemia in SCI have been posited, but have all assumed a causative link to altered skeletal muscle. Duckworth and Elder offered a potential connection between DM and replacement of skeletal muscle with adipose tissue, while Bauman theorized that the post-prandial hyperglycemia of SCI could be related to structural changes in denervated muscle. While these ideas are partly supported by experimental findings, none has been proven, and other potential explanations remain unexplored.

Several authors have demonstrated complicated relationships between upper gastrointestinal motility and glucose regulation. Specifically, rapid gastric emptying seems to be associated with elevated post-prandial serum glucose levels, and suppression of duodenal flow events attenuates small bowel glucose absorption and post-prandial hyperglycemia $[4,5]$. While no authors to our knowledge have evaluated objective physiologic measures of gut motility and function in SCI, we hypothesized that the "hyper-reflexic" high-tone bowel that often accompanies upper motor neuron injuries [6] may also alter glucose uptake from the bowel.

In this article, we report preliminary data evaluating the effects of severity of neurogenic bowel on disordered glucose metabolism in individuals with chronic SCI. We sought to demonstrate that severity of neurogenic bowel would positively correlate with serum glucose and insulin levels on OGTT, hoping to then explore potential explanatory mechanisms.

\section{Materials and Methods}

Data were collected from 19 adults with SCI and 10 without, all of whom responded to informational brochures distributed through
Phys Med Rehabil Int - Volume 4 Issue 2 - 2017 ISSN : 2471-0377 | www.austinpublishing group.com Stillman et al. (c) All rights are reserved
Citation: Stillman M, Graves D, Lenneman C and Williams S. Neurogenic Bowel, Disordered Glycemic Control and Chronic Spinal Cord Injury: A Preliminary Investigation. Phys Med Rehabil Int. 2017; 4(2): 1113. 
Table 1: Comparison of glucose and insulin levels between injury groups during the oral glucose tolerance test. A $2 \mathrm{hr}$ glucose between 140 and $200 \mathrm{mg} / \mathrm{dL}$ indicates IGT, while a value of $200 \mathrm{mg} / \mathrm{dL}$ or higher indicates DM.

\begin{tabular}{|c|c|c|c|c|}
\hline & Fasting & $\mathbf{3 0}$ Min & 60 Min & 120 Min \\
\hline \multicolumn{5}{|c|}{ Glucose (mg/dL) } \\
\hline $\mathrm{SCl}(\mathrm{n}=19)$ & 81.32 & 140.26 & $142.11^{\star}$ & $125.47^{*}$ \\
\hline $\mathrm{Non}-\mathrm{SCl}(\mathrm{n}=10)$ & 87.4 & 122.3 & $107.40^{\star}$ & $83.00^{\star}$ \\
\hline \multicolumn{5}{|c|}{ Insulin (ulU/mL) } \\
\hline $\mathrm{SCl}(\mathrm{n}=19)$ & 7.86 & $77.87^{*}$ & $104.11^{*}$ & $87.45^{\star}$ \\
\hline $\mathrm{Non}-\mathrm{SCl}(\mathrm{n}=10)$ & 6.37 & $43.03^{*}$ & $43.91^{*}$ & $20.07^{*}$ \\
\hline
\end{tabular}

*indicates a significant difference at .05 .

Table 2: Comparison of glucose and insulin levels by level and completeness of injury groups during the oral glucose tolerance test. A $2 \mathrm{hr}$ glucose between 140 and $200 \mathrm{mg} / \mathrm{dL}$ indicates IGT, while a value of $200 \mathrm{mg} / \mathrm{dL}$ or higher indicates DM.

\begin{tabular}{|c|c|c|c|c|}
\hline & Fasting & $\mathbf{3 0}$ Min & $\mathbf{6 0}$ Min & $\mathbf{1 2 0}$ Min \\
\hline \multicolumn{5}{|c|}{ Glucose $\mathbf{( m g / d L})$} \\
\hline Paraplegic $(n=9)$ & 80.74 & 129.55 & 120.11 & 111.44 \\
\hline Tetraplegic $(n=10)$ & 80.8 & 149.9 & 161.9 & 138.1 \\
\hline Complete $(n=14)$ & 80.28 & 139.07 & 132.79 & 120.92 \\
\hline Incomplete $(n=5)$ & 84.2 & 143.6 & 168.2 & 138.2 \\
\hline & Insulin (ulU/mL) & \\
\hline Paraplegic & 9.45 & 75.1 & 89.93 & 79.35 \\
\hline Tetraplegic & 6.59 & 80.1 & 115.46 & 93.93 \\
\hline Complete & 7.53 & 74 & 81.85 & 80.94 \\
\hline Incomplete & 8.72 & 87.94 & 162 & 104.36 \\
\hline
\end{tabular}

*indicates a significant difference at .05.

an outpatient rehabilitation facility in Louisville, KY. As this work represents a subset analysis of a study investigating fecal bacterial patterns and systemic inflammatory markers in individuals with SCI, inclusion criteria were relatively broad but exclusion criteria were quite specific. Those eligible had to have sustained an injury over 1 year prior to enrollment (for those with SCI) and were between the ages of 18 and 75. Exclusion criteria included: i) being pregnant or lactating; ii) taking probiotics, prebiotics, or non-topical steroid medications; iii) smoking; iv) having been diagnosed with or treated for DM; v) having taken systemic antibiotics or investigational drugs within one month of enrollment; vi) having a significant acute or chronic infection; vii) active psychosis or substance abuse; viii) having had a myocardial infarction or coronary artery bypass grafting within the previous 6 months; and ix) having known severe cardiopulmonary, hepatic, renal, or cerebrovascular disease.

Once screened and consented, participants were administered Krough et al's validated neurogenic bowel questionnaire [7] by which individuals may be assigned designations of "very minor" (score 0-6), "minor" (score 7-9), "moderate" (score of 10-13), or "severe" (score of 14 or higher) bowel dysfunction. They were then assigned scores and severity classifications and underwent 2 hour OGTTs (fasting samples followed by collections at 30, 60, and 120 minutes) and testing for hemoglobin A1c (HbAlc). All study procedures were approved by the University of Louisville Institutional Review Board.

Data for the glucose and insulin levels were analyzed using repeated measures general linear models (GLM) using an alpha level
Table 3: Comparison of glucose and insulin levels for $\mathrm{SCl}$ cases based on neurogenic bowel scores. A score of 14 or higher indicates severe bowel dysfunction.

\begin{tabular}{|c|c|c|c|c|}
\hline & Fasting & 30 Min & 60 Min & 120 Min \\
\hline \multicolumn{5}{|c|}{ Glucose (mg/dL) } \\
\hline 14 or higher & 78.44 & 143.56 & 143.11 & 137.78 \\
\hline 13 or lower & 83.9 & 137.3 & 141.2 & 114.4 \\
\hline \multicolumn{5}{|c|}{ Insulin (ulU/mL) } \\
\hline 14 or higher & 6.73 & 68.2 & 88.55 & 99.54 \\
\hline 13 or lower & 8.98 & 87.55 & 119.67 & 75.35 \\
\hline
\end{tabular}

of .05 for all analyses. Demographic and classification data were analyzed using independent groups t-test and Pearson correlation coefficient.

\section{Results}

Among 19 participants with SCI, 37\% $(n=7)$ were female, average age was 50.9 years (range 25-70), average duration of injury was 11.37 years (range 1-45), and average bowel score was 11.84 (range 2-19). Ten of the $19(52.6 \%)$ had tetraplegia, and $14(73.7 \%)$ had motor complete (AIS A or B) injuries. Among the 10 non-injured controls, half were female and average age was 43.3 years.

During OGTT, mean glucose and insulin levels were significantly higher among subjects with SCI than those without (Table 1), though HbA1clevels in the two groups were nearly identical (5.52 for those with SCI vs 5.56 for controls). There were no significant differences in $\mathrm{HbA1c}$, glucose, or insulin levels between subjects with complete or incomplete injuries or between those with paraplegia or tetraplegia (Table 2). Additionally, there were no significant differences between the bowel scores of those with motor complete and motor incomplete injuries $\left(\mathrm{X}^{2}=16.42, \mathrm{df}=12, \mathrm{p}=.173\right)$ or tetraplegia versus paraplegia $\left(\mathrm{X}^{2}=14.22, \mathrm{df}=12, \mathrm{p}=.281\right)$. Four participants with SCI had 120 minute glucose levels between 140 and 200 (criteria for IGT), while 1 had a level over 200 (criteria for DM).

Subjects with SCI were divided into those with severe neurogenic bowel (score of 14 or greater; $n=9$ ) and those with moderate, mild, or very mild dysfunction (score $<13 ; n=10$ ). There was no significant association of the bowel severity grouping with either level or motor completeness of injury. Those with severe neurogenic bowel had nonsignificant elevations of both insulin and glucose levels at 120 minutes (137.78 vs 114.4 for glucose; 99.54 vs 75.35 for insulin) (Table 3), though there were no differences between the two groups in HbAlc (5.67 for severe dysfunction vs 5.36 for others) or fasting insulin and glucose levels.

Subjects with SCI were also separated into those with normal $(<5.7 ; \mathrm{n}=13)$ and elevated $(>5.7 ; \mathrm{n}=6) \mathrm{HbA1c}$ levels. There was no correlation between $\mathrm{HbA} 1 \mathrm{c}$ and fasting or 120 minute glucose or insulin levels in either cohort.

\section{Discussion}

While the significance of between group (SCI vs non-injured) and within group (SCI cohort) comparisons was limited by both small sample sizes and large variances, these preliminary data raise several important questions. 
First, while our OGTT results mirror Bauman's, demonstrating higher glucose and insulin levels among participants with SCI, they also suggest a possible correlation between gut dysfunction, insulin insensitivity, and post-prandial hyperglycemia in people with chronic injuries. In our cohort of subjects with SCI, those with severe neurogenic bowel had non-significant though $23 \mathrm{mg} / \mathrm{dL}$ glucose elevations at 120 minutes, consistent with an insulin resistant state.

There is a growing awareness of ties between gastrointestinal function, systemic inflammation, and altered glucose metabolism, though they have yet to be elucidated in individuals with SCI. Cani et al [8] demonstrated that lipopolysaccharide, a component of certain gram negative bacterial membranes, can translocation across a "leaky" gut wall, stimulating a cascade of inflammatory cytokine release, increased adiposity, and hyperglycemia. Gungor et al [9] recently described fecal microbiomal shifts among individuals with SCI resulting in reduced production of butyrate, a fatty acid instrumental in preserving gut barrier functions [10]. While our data and the scope of this preliminary work are insufficient to support conclusions about metabolic effects of neurogenic bowel, they raise the possibility of a unifying explanation (microbiomal perturbations with resulting "leaky" gut) for both the adipose deposition in skeletal muscle and the post-prandial hyperglycemia often seen in people with SCI. Broader investigations with larger sample sizes are certainly called for.

Second, our results may challenge previously described rates of IGT and DM among people with SCI. While Duckworth and Bauman found high rates of these diseases among their cohorts of veterans, only $21 \%$ of our subjects with injuries had IGT and $5 \%$ had DM. Our prevalence rate of IGT is higher than the national average (11\% for IGT and $9.3 \%$ for DM) [11], yet our data raise the possibility that studies exclusively enrolling veterans seeking care within the Veterans Administration system are inherently biased, and that further investigation of non-veteran cohorts will yield more accurate and representative data.

Third, this study raises the question of how best to screen people with SCI for DM, a disease that is treatable if detected but that is accompanied by significant microvascular and macrovascular complications. Bauman demonstrated that fasting glucose levels do not accurately reflect individuals' with SCI ability to metabolize glucose (ie: normal fasting sugar levels with "failure" of OGTT), and our data revealed no correlation between subjects' HbAlc levels and performance on OGTT. The two tests used in general practice to screen patients for DM do not, in the setting of SCI, seem to predict performance on the "gold standard" glucose tolerance test. The finding of normal fasting glucose levels among our participants with SCI suggests that chronic injury is not associated with a "defense" of elevated glucose levels. That is, that the hyperglycemia of chronic SCI is solely post-prandial, hence not associated with excessive red blood cell glycolation. If this is so, HbAlc ought to be considered a reliable screening test for DM for individuals with SCI. Future studies are needed to correlate $\mathrm{HbAlc}$ and OGTT results with pancreatic function and indicators of vascular health in subjects with chronic injuries.

\section{Conclusion}

These results, while limited due to sample size and variance, suggest the possibility that the neurogenic bowel of chronic SCI may be linked to disordered glucose regulation. More and larger studies are needed to explore whether this association holds.

\section{References}

1. Duckworth WC, Solomon SS, Jallepalli P, Heckemeyer C, Finnern J, Powers A. Glucose intolerance due to insulin resistance in patients with spinal cord injuries. Diabetes. 1980; 29: 906-910.

2. Bauman WA, Spungen AM. Disorders of carbohydrate and lipid metabolism in veterans with paraplegia or quadriplegia: a model of premature aging. Metabolism. 1994; 43: 749-756.

3. Elder CP, Apple DF, Bickel CS, Meyer RA, Dudley GA. Intramuscular fat and glucose tolerance after spinal cord injury--a cross-sectional study. Spinal Cord. 2004; 42: 711-716.

4. Chaikomin R, Wu KL, Doran S, Jones KL, Smout AJ, Renooij W, et al. Concurrent duodenal manometric and impedance recording to evaluate the effects of hyoscine on motility and flow events, glucose absorption, and incretin release. Am J Physiol Gastrointest Liver Physiol. 2007; 292: G10991104.

5. Jones KL, Horowitz M, Carney BI, Wishart JM, Guha S, Green L. Gastric emptying in early noninsulin-dependent diabetes mellitus. J Nucl Med. 1996; 37: 1643-1648.

6. Krassioukov A, Eng JJ, Claxton G, Sakakibara BM, Shum S. Neurogenic bowel management after spinal cord injury: a systematic review of the evidence. Spinal Cord. 2010; 48: 718-733.

7. Krogh K, Christensen P, Sabroe S, Laurberg S. Neurogenic bowel dysfunction score. Spinal Cord. 2006; 44: 625-631.

8. Cani PD, Amar J, Iglesias MA, Poggi M, Knauf C, Bastelica D, et al. Metabolic endotoxemia initiates obesity and insulin resistance. Diabetes. 2007; 56: 1761-1772.

9. Gungor B, Adiguzel E, Gursel I, Yilmaz B, Gursel M. Intestinal Microbiota in Patients with Spinal Cord Injury. PLoS One. 2016; 11: e0145878.

10. Riviere A, Selak M, Lantin D, Leroy F, De Vuyst L. Bifidobacteria and ButyrateProducing Colon Bacteria: Importance and Strategies for Their Stimulation in the Human Gut. Front Microbiol. 2016; 7: 979.

11. Harris MI, Flegal KM, Cowie CC, Eberhardt MS, Goldstein DE, Little RR, et al. Prevalence of diabetes, impaired fasting glucose, and impaired glucose tolerance in U.S. adults. The Third National Health and Nutrition Examination Survey, 1988-1994. Diabetes Care. 1998; 21: 518-524.
Phys Med Rehabil Int - Volume 4 Issue 2 - 2017
ISSN : 2471-0377 | www.austinpublishing group.com
Stillman et al. @ All rights are reserved

Submit your Manuscript | www.austinpublishing group.com
Citation: Stillman M, Graves D, Lenneman C and Williams S. Neurogenic Bowel, Disordered Glycemic Control and Chronic Spinal Cord Injury: A Preliminary Investigation. Phys Med Rehabil Int. 2017; 4(2): 1113. 